\title{
Locomotor Brachialis: A clinical peep into atherosclerosis and hypertension mediated organ damage in the elderly
}

\author{
Thirunavukarasu Kumanan and Nadarajah Sreeharan \\ Faculty of Medicine, Jaffna, Sri Lanka
}

Over the years, clinical practice has been transformed by the advent of sophisticated diagnostic tools. Although their benefits are obvious, they have resulted in neglect of valuable bedside physical signs which adorned clinical practice over centuries. A single pathognomonic physical sign could often lead to a diagnosis without the need of expensive investigations. Locomotor brachialis (LB) is one such neglected physical sign often observed in the elderly. It refers to a dilated, thickened, tortuous and pulsatile brachial artery, usually associated with atherosclerosis and sustained hypertension. It is easily visible while recording the blood pressure due to the superficial location of the brachial artery and the atrophy of adjacent muscle and soft tissue mass with advancing age.

The brachial artery is categorized as a medium sized central artery along with other vital vessels like the coronary, cerebral and renal arteries. Stiffness of the brachial artery can therefore be a good predictor of future more serious cardiovascular and renal events. Sorenson et al[1] concluded that atherosclerosis in the brachial artery is significantly correlated with both coronary and carotid disease atherosclerosis even in younger adults, and that LB could serve as a "clinical surrogate" for more general atherosclerosis across all age groups.

\section{Keywords: Locomotor brachialis}

\section{Author responsible for correspondence: \\ Dr. Thirunavukarasu Kumanan \\ Faculty of Medicine Jaffna \\ Email: tkumanan@univ.jfn.ac.lk}

https://orcid.org/0000-0001-5735-4313

DOI: http://doi.org/10.4038/cjms.v56i1.4960
Arterial stiffness is one of the earliest detectable signs of functional and structural changes in the vascular wall[2]. Thus, assessment of central artery stiffness has become a focal point in early detection of vascular diseases. Several investigations, including brachial ankle pulse wave velocity (baPWV), flow mediated dilation (FMG) and intimal media thickness (IMT) have been developed to study vessel wall thickness and impedance. There is mounting evidence to suggest that baPWV could predict future cardiovascular events and total mortality[3]. Impaired FMG of the brachial artery is also a predictor of future cardiovascular events and has been shown to have a similar prognostic accuracy as traditional cardiovascular risk stratification scores in older adults[4]. IMT of the brachial artery may be correlated with the grade of atherosclerosis and could also be used as a marker of vascular function, providing additive information for risk stratification of subjects with cardiovascular risk factors[5].

It is reasonable to assume that the changes in the brachial artery are a visual representation of changes of clinical significance in other hidden internal arteries; the assessment of which need sophisticated investigations that may not be readily available in primary care systems of low middleincome countries. However, LB could act as an easily available "red flag" for any potential damage to a patient's vasculature, thus alerting primary care physicians to seek specialist consultation for more targeted investigations and risk assessments. We feel that this vital clinical sign should receive more emphasis in the clinical training of undergraduates and primary care professionals. Further research is also needed to clinically grade LB and validate its presence as a marker for vascular disease with adverse outcomes. 


\section{References}

1. Sorensen KE, Kristensen IB, Celermajer DS. Atherosclerosis in the human brachial artery. J Am Coll Cardiol. United States; $1997 \mathrm{Feb}$;29(2):318-22.

2. Cohn JN, Quyyumi AA, Hollenberg NK et al. Surrogate markers for cardiovascular disease: functional markers. Circulation. United States; 2004 Jun;109(25 Suppl 1):IV31-46.

3. Sugawara J, Tanaka H. Brachial-Ankle Pulse Wave Velocity: Myths, Misconceptions, and Realities. Pulse (Basel, Switzerland). 2015 Sep;3(2):10613.

4. Yeboah J, Crouse JR, Hsu F-C et al. Brachial flow-mediated dilation predicts incident cardiovascular events in older adults: The Cardiovascular Health Study. Circulation. United States; 2007 May;115(18):2390-7.

5. Iwamoto $\mathrm{Y}$, Maruhashi $\mathrm{T}$, Fujii $\mathrm{Y}$ et al. Intima-media thickness of brachial artery, vascular function, and cardiovascular risk factors. Arterioscler Thromb Vasc Biol. United States; 2012 Sep;32(9):2295-303. 